\title{
Maternal and Fetal Outcomes in Emergency versus Elective Cesarean Sections at a Tertiary Healthcare Setting in Southern India: A Prospective Observational Study
}

\author{
${ }^{1}$ Garima Nag, ${ }^{2}$ Padmalatha VV, ${ }^{3}$ Shubha Rama Rao
}

\begin{abstract}
Introduction: The present study was conducted to evaluate the maternal and fetal outcomes in elective versus emergency cesarean section (CS), performed at a tertiary hospital in southern India.
\end{abstract}

Materials and methods: The study was a prospective observational study conducted at a tertiary referral center in Bangaluru, India. 500 consecutive CS, over 29 months (January 2011 to June 2013), were studied. The questionnaire-based tool was used to collect data from the patient's case sheet, labor record, intra-operative records, and treating clinician. The main outcomes were maternal and early neonatal ( $<7$ days) severe morbidity.

Results: Total of 3393 deliveries took place during the study period. The CS rate was $16 \%$. The emergency CS was $58.4 \%$ of all sections. In the emergency CS group, 89 patients (30.4\%) experienced at least one intra-operative difficulty/complication against 54 patients $(25.9 \%)$ in the elective CS group $(p=0.31)$. The incidence of any postoperative complication was $30.3 \%$ and $24.3 \%$, in the elective and emergency CS group, respectively $(p=0.20)$. The mean (SD) length of hospital stay was 4.57 (1.8) and 4.7 (1.2) days in the elective and emergency CS group, respectively $(p=0.30)$. There was no maternal mortality. In the emergency CS group, neonates tended to have significantly lower birth weight, APGAR scores at 1 and 5 minutes, had higher morbidity and required NICU more frequently.

Conclusion: Although maternal morbidity was similar between the emergency and elective CS patients, the fetal outcomes were worse in the emergency CS patients. Whether this is due to fetal distress or complication as an indication for emergency CS or the result of emergency CS is not clear and could be evaluated in future studies.

Keywords: Elective cesarean section, Emergency cesarean section, Maternal morbidity, Neonatal morbidity.

How to cite this article: Nag G, Padmalatha VV, Rao SR. Maternal and Fetal Outcomes in Emergency versus Elective Cesarean Sections at a Tertiary Healthcare Setting in Southern India: A Prospective Observational Study. J South Asian Feder Obst Gynae 2018;10(Suppl 2):413-418.

\footnotetext{
${ }^{1}$ Registrar, ${ }^{2}$ Consultant, ${ }^{3} \mathrm{Head}$

${ }^{1}$ Department of Emergency Medicine, Flinders Medical Centre, Adelaide, Australia

${ }^{2,3}$ Department of Obstetrics and Gynaecology, St Martha's Hospital, Benagluru, Karnataka, India

Corresponding Author: Garima Nag, Registrar, Department of Emergency Medicine, Flinders Medical Centre, Adelaide, Australia, e-mail: garima.prak@gmail.com
}

Source of support: Nil

Conflict of interest: None

Date of received: 9 June 2017

Date of acceptance: 14 May 2018

Date of publication: July 2019

\section{INTRODUCTION}

Cesarean section (CS) was once deemed to be a high-risk procedure with high mortality, hemorrhage and infection rates. However, in the modern era, CS is regarded as a safe procedure and consequently, there has been a dramatic increase in the frequency of CS rates around the world. ${ }^{1}$ Well recognized indications for elective CS include marked contracted pelvis, mild disproportion associated with complicated factors such as elderly primigravida, preeclampsia, eclampsia, intrauterine growth restriction, central placenta previa, and other conditions of placental insufficiency. But due to the increasing safety profile of this procedure, the reported indications have grown to include circumstances where conventionally, vaginal delivery would be considered as a safe option. ${ }^{2}$ Use of aseptic techniques, antibiotics, blood transfusions, and modern anesthetic techniques are some of the reasons behind the decreasing morbidity and mortality associated with this procedure. ${ }^{3}$

There is also evidence, however, that morbidity of CS may be higher than vaginal birth. ${ }^{4}$ Additionally, the outcomes of CS such as morbidity may be strongly influenced by whether it has been performed as an elective or emergency procedure. ${ }^{5}$ Emergency CS is commonly performed for fetal distress, prolonged/obstructed labor, severe preeclampsia, antepartum hemorrhage, and ruptured uterus. Other than circumstances where there are absolute indications for CS, there may be a benefit of trialing vaginal delivery. However, the downside is that this approach may be associated with a higher incidence of emergency CS. If the outcomes of emergency and elective CS are somewhat similar, then the benefit of the above strategy of trialing vaginal delivery and attempting CS only when it fails may be justified. However, the outcomes of emergency CS may vary considerably in different contexts, depending upon factors such as 
preventive measures taken, facilities and availability of skilled staff. ${ }^{2,3}$ Also, the maternal ante-natal complications, co-morbidities and care, and fetal condition in these circumstances are likely to impact the outcome. Hence there is value in comparing outcomes of emergency versus elective CS in different healthcare settings, to better inform the approach to delivery in the absence of absolute indications for elective CS.

The present study was conducted to evaluate the maternal, and fetal outcomes in elective versus emergency CS performed at a tertiary hospital in southern India.

\section{MATERIALS AND METHODS}

\section{Setting}

The study was a prospective observational study conducted at St. Marthas Hospital, Bengaluru, India. This is a teaching, tertiary level, 534 bedded referral center, which caters to 2,50,000 outpatients and 21,000 inpatients annually. For this study, 500 consecutive CS, over 29 months (January 2011 to June 2013), were studied. The patients who are posted for elective CS are admitted the night before. All patients who were posted for emergency CS had CTG monitoring, except for those who presented to the emergency department with complications such as cord prolapse and were taken urgently to the operating theatre. The Labor ward, operating theatre, and emergency department are close to each other. The hospital has adult and pediatric intensive care services.

The study was ethically conducted in accordance with the 'Declaration of Helsinki' and was approved by the institutional ethics committee.

\section{Inclusion and Exclusion Criteria}

Patients were screened for enrollment in the study, once they underwent CS. Patients were excluded if the CS was performed after rupturing of the uterus or in less than 28 weeks of gestation.

\section{Definitions}

Cesarean deliveries were classified as elective if the operation was decided by the specialist before the onset of labor. Cesarean deliveries were classified as an emergency when the patients were admitted in labor, as judged by the specialist on duty (regular contractions with cervical dilatation) or the CS was not pre-planned/scheduled and there was the concern of impending feto-maternal compromise. Patient having less than three antenatal visits in our hospital or patients with irregular antenatal check-up outside were classified as 'unbooked'. Difficulty in extraction was considered in cases requiring forceps for extraction, Patwardhan's or modified Patwardhan's ${ }^{6}$ technique for extraction of the baby.
Fever was defined as the rise of temperature to $100.4^{\circ} \mathrm{F}$ or $38^{\circ} \mathrm{C}$ or more on two separate occasions, 24 hours apart. Urinary tract infection was evident by the presence of pus cells in urine routine microscopic examination or bacterial growth on urine culture. For this study, the presence of moderate to severe pain was recorded and was defined as score $\geq 4$ on the numeric visual analog scale. ${ }^{7}$

Severe maternal morbidity was defined as any one of the following: hemorrhage requiring hysterectomy or transfusion, uterine rupture, anesthetic complications, shock, cardiac arrest, acute renal failure, assisted ventilation, venous thromboembolic event, sepsis, in-hospital wound disruption, wound hematoma, or both. ${ }^{4}$ Neonatal morbidity was defined as any of the following: Respiratory morbidity such as transient tachypnea of the newborn (TTN) or respiratory distress syndrome (RDS); NICU admission of more than 24 hours, and APGAR score at 5 minutes of $\leq 6$.

Maternal mortality was defined as death within 42 days of delivery, and perinatal mortality was defined as death (fetal/neonatal) within 28 days after birth. ${ }^{8}$

\section{Data Collection}

The questionnaire-based tool was used to collect data from the patient's case sheet, labor record, intraoperative records, and treating clinician. To prevent bias, the person collecting data was unaware of the objectives of the study.

The following data were recorded:

- Patient characteristics: Age, GPL data (gravida, para, living), number of previous CS, previous obstetric complications, ante-natal history (number of antenatal visits, complications during current gestation, duration of gestation) and delivery data such as indication, type (emergency/elective) and duration of CS, time of operation,type of anesthesia, difficulty in opening the peritoneum, difficulty in approaching the lower segment, presence of adhesions, mode of delivery of the baby, difficulty and complications in the delivery of the baby, amount of bleeding, extension of uterine incision, state of uterus after removal of placenta, was noted. Amount of blood loss was estimated by the visual method of checking, taking into account the number of mops, drapes used and measuring the contents of the suction bag.

- Postoperative complications: Pain, fever, urinary tract infection, wound infection or gaping was noted. The pain was a subjective variable recorded on a standard numeric visual analog rating scale. Any serous or purulent discharge from the wound and obvious wound gaping was noted as postoperative complications. Admission to intensive care, mortality, and duration of hospital stay (days) was recorded. 
- Fetal outcomes: Mortality, weight, APGAR score (1 minute and 5 minutes), TTN or RDS and admission to neonatal intensive care for $>24$ hours were recorded.

- Outcomes: The main outcomes were maternal and early neonatal ( $<7$ days) severe morbidity.

\section{Statistical Analysis}

Based on average (urban and rural) CS rate of $15 \%$ in Karnataka state, ${ }^{9}$ we estimated the minimum sample size of 196 subjects in each study arm. The sample size estimation assumed $95 \%$ confidence interval and $5 \%$ precision and used the following formula: , where $\mathrm{Z}$ is the value from a standard normal distribution, $\mathrm{e}$ is the desired precision and $\mathrm{P}$ is the expected proportion.

The data has been presented using tabular and graphical aids. Both inferential and descriptive statistics have been used. Proportions have been presented as percentages and continuous variables have been described using mean (standard deviation). Proportions were compared using the Chi-square test, while continuous variables were compared using the Mann-Whitney $U$ test or students T-test depending upon normality of distribution. The analysis was performed using statistical package for social sciences (SPSS) version 21. For all tests, a two-sided $p$ value of less than 0.05 was considered significant.

\section{RESULTS}

Total of 3393 deliveries took place during the study period. The CS rate was $16 \%$ during this time. The emergency CS was $58.4 \%$ of all sections. The baseline demographics of patients are given in Table 1. There were more pre-term pregnancies and primigravida patients with higher body mass index (BMI), amongst the emergency CS group as compared to elective CS group. All patients with a history of previous CS had only one CS except for three patients, who had two previous CS. Nonreassuring fetal heart rate (NRFHR) $(33.1 \%)$, and failure to progress $(30.3 \%)$ were the most frequent indications for previous CS. Of the 461 patients who had ANC follow-up and were booked patients, no risk factors were identified in $233(50.5 \%)$ patients. Common risk factors that were identified, included preeclampsia $(18.1 \%)$, severe preeclampsia (17.4\%), gestational diabetes mellitus (12.8\%), multi-foetal pregnancy (9.6\%), poor follow-up (5\%), chronic hypertension ( $4 \%)$ and placenta previa $(2.8 \%)$.

Various indications of CS are listed in Table 2. Amongst the patients undergoing elective $\mathrm{CS}$, the majority had 'previous $C S^{\prime}$ ' as the indication; however, amongst the patients undergoing emergency CS, the majority had NRFHR as the indication. The second most common indication for elective CS was 'mal-presentation', while in an emergency CS group it was 'failure to progress'. Within the 'other indications', the most common amongst the elective CS was maternal request $(50 \%)$; while cord prolapse $(60 \%)$, eclampsia (30\%) and fetal growth retardation (25\%) were the common 'other indications' in emergency CS.

Overall, 471 (94.2\%) patients received spinal anesthesia while only $29(5.8 \%)$ patients received general anesthesia (GA). All GA cases were emergency CS and accounted for only $10 \%$ of all emergency CS cases. Features of the intraoperative course of patients are listed in Table 3. In the emergency CS group, 89 patients (30.4\%) experienced at least one intraoperative difficulty / complication against 54 patients (25.9\%) in the elective CS group (Chi-square test $p=0.31$ ). The most frequent difficulty/ complication seen during emergency CS was peritoneal adhesions, which was also the case with elective CS. Of all the patients requiring CS, $11(2.2 \%)$ had atonic

Table 1: The baseline demographics of patients

\begin{tabular}{llll}
\hline & Emergency CS $(n=292)$ & Elective CS $(n=208)$ & $p$ value \\
\hline Age $($ years $)$ & $26(4.6)$ & $26(3.6)$ & NS \\
BMI $\left(\mathrm{kg} / \mathrm{m}^{2}\right)$ & $30(4)$ & $28.6(4.2)$ & 0.002 \\
Height $(\mathrm{cm})$ & $152(4.9)$ & $154.3(4.3)$ & 0.001 \\
Weight $(\mathrm{kg})$ & $69(10)$ & $68.7(11)$ & $\mathrm{NS}$ \\
Primigravida & $169(57.9 \%)$ & $93(44.7 \%)$ & 0.005 \\
\hline Term & & $2(0.8 \%)$ & $<0.001$ \\
\hline Early preterm $(<34$ weeks) & $2(0.8 \%)$ & $4(2 \%)$ & $\mathrm{NS}$ \\
Late preterm $(34-37$ weeks) & $53(18 \%)$ & $202(97.2 \%)$ & $<0.001$ \\
Term $(>37$ weeks) & $237(81.2 \%)$ & $38(2.5)$ & $<0.001$ \\
Duration of gestation (weeks) & $38.5(3.5)$ & $50(38.1 \%)$ & 0.04 \\
History of previous abortions & $82(61.9 \%)$ & $88(67 \%)$ & $198(95.2 \%)$ \\
History of previous cesarean section & $43(33.1 \%)$ & & \\
Antenatal checkup-booked cases & $263(90 \%)$ & & \\
\hline
\end{tabular}

BMI, body mass index 
postpartum hemorrhage post-section. Of these, 9 patients were in the elective CS group and 2 patients were in the emergency CS group. The amount of blood loss in patients with atonic postpartum hemorrhage was three times the average blood loss, i.e., $916 \mathrm{~mL}$.

Postoperative complications occurred in 134 (26.8\%) patients. The incidence of any postoperative complication in the elective CS group was 30.3\%, and in the emergency CS group, it was $24.3 \%(p=0.20)$ (Table 4 ). The mean (SD) length of hospital stay for patients was 4.64 (1.47) days, which ranged from 3 days to 15 days. The mean (SD) length of hospital stay in the elective CS group was 4.57 (1.8) days which was similar to 4.7 (1.2) days in the emergency CS (students t-test, $p=0.3$ ). There was no maternal mortality.

In contrast to maternal morbidity, neonatal morbidity was significantly different between the emergency and elective CS groups (Table 5). In the emergency CS group, neonates tended to have significantly lower birth weight,

Table 2: Prevalence (\%) of various indications of cesarean section in the study

\begin{tabular}{lll}
\hline & $\begin{array}{l}\text { Emergency CS } \\
(n=292)\end{array}$ & $\begin{array}{l}\text { Elective CS } \\
(n=208)\end{array}$ \\
\hline Previous CS & $9(3 \%)$ & $86(41 \%)$ \\
Mal-presentation & $23(8 \%)$ & $54(26 \%)$ \\
Placenta previa & 0 & $8(4 \%)$ \\
CPD & $11(4 \%)$ & $8(4 \%)$ \\
Failure to & $35(12 \%)$ & 0 \\
progress & $120(41 \%)$ & 0 \\
NRFHR & $9(3 \%)$ & 0 \\
APH & $9(3 \%)$ & $2(1 \%)$ \\
Multifetal & & 0 \\
pregnancy & $26(9 \%)$ & $50(24 \%)$ \\
MSL & $50(17 \%)$ &
\end{tabular}

CS, cesarean section; CPD, cephalo-pelvic disproportion; NRFHR, nonreassuring fetal heart rate; $\mathrm{APH}$, antepartum hemorrhage; MSL, meconium stained liquor
APGAR scores at 1 and 5 minutes, had higher morbidity and required NICU more frequently.

\section{DISCUSSION}

Our study demonstrated that the maternal morbidity was similar, whether the CS was performed selectively or on an emergency basis. The neonatal morbidity, although minimal, was significantly worse in emergency CS as compared to elective CS.

There is the heterogeneity of results reported by different studies in the literature. ${ }^{10-16}$ The age of our study population is similar to previously reported studies; ${ }^{10-14}$ however, there is a lack of data on BMI of patients. Patients who underwent emergency CS tended to have higher BMI. Obesity is known to be associated with more complications such as GDM, preeclampsia, and macrosomia which may increase the risk of CS. The CS rate at our hospital was less than other studies in Indian setup despite being an urban tertiary level referral center. ${ }^{12-14}$ This may be due to close follow-up of patients, education, and impetus for the trial of vaginal delivery. This is represented by the fact that over $90 \%$ of cases had an ante-natal checkup. This is in contrast to poor rates on ante-natal follow-up reported in the emergency CS group in previous studies. ${ }^{15,16}$ However, similar to these studies, statistically, a lesser proportion of patients in the emergency CS had an ante-natal check-up. The frequent indications for CS in the emergency CS group were NRFHR and failure to progress, which is similar to previous studies; however other indications such as eclampsia and preeclampsia were significantly more in the previous study, ${ }^{12}$ which may explain the feto-maternal outcomes worse than our study. Similar to other studies the common indications for elective CS in our study were previous CS and malpresentation.

The maternal morbidity and mortality in our study were significantly better than other studies ${ }^{12}$ and also it did not differ between the emergency and elective CS group. This highlights the importance of context when

Table 3: Intraoperative course in emergency and elective CS

\begin{tabular}{llll}
\hline & Emergency CS & Elective CS & $p$ value \\
\hline Duration (minutes) & $54.2(10.57)$ & $54.6(12.7)$ & 0.7 \\
Blood loss (mL) & $355.6(146.2)$ & $323(125)$ & 0.01 \\
Patients requiring blood transfusion & $14(4.8 \%)$ & $8(3.8)$ & NS \\
\hline Difficulties/complications & & & - \\
Dissection of rectus sheath & $9(8.9 \%)$ & $18(20.7 \%)$ & - \\
Peritoneal adhesions & $38(37.6 \%)$ & $39(44.8 \%)$ & - \\
Difficult extraction & $27(26.7 \%)$ & $5(5.7 \%)$ & - \\
Advancement of bladder & $18(17.8 \%)$ & $18(20.7 \%)$ & - \\
Extension & $4(3.9 \%)$ & $3(3.4 \%)$ & - \\
Bladder injury & $2(1.9 \%)$ & $1(1.1 \%)$ & - \\
Other & $3(2.9 \%)$ & $3(3.4 \%)$ & \\
\hline
\end{tabular}


Maternal and Fetal Outcomes in Emergency versus Elective Cesarean Sections at a Tertiary Healthcare Setting

\begin{tabular}{lll}
\hline \multicolumn{3}{c}{ Table 4: Postoperative complications } \\
\hline & Emergency & Elective \\
& CS & CS \\
\hline Pain (score $\geq 4)$ & $37(45.1 \%)$ & $27(49.1 \%)$ \\
Fever & $15(18.3 \%)$ & $8(14.5 \%)$ \\
Urinary tract infection & $10(12.2 \%)$ & $9(16.4 \%)$ \\
Wound infection/gaping & $7(8.5 \%)$ & $5(9.1 \%)$ \\
Admission to intensive care unit & $3(3.6 \%)$ & 0 \\
Others & $10(12.2 \%)$ & $6(10.9 \%)$ \\
\hline
\end{tabular}

There was no significant differences between groups

assessing these outcomes. Najam et al. ${ }^{12}$ compared the outcomes of emergency and elective CS on only 177 cases in northern India. Although not subjected to statistical analysis, the perinatal morbidity was relatively higher $(84 \%$ vs $16 \%)$ in the emergency CS group. Another study from Nepal, ${ }_{11}^{11}$ compared outcomes across $254 \mathrm{CS}$; however, the number of emergency CS was much lower than our study. Similar to Najam et al., ${ }^{12}$ they found higher morbidity in emergency CS group reflected by a higher incidence of the length of stay, fever, UTI and wound infection rates. The details of Antenatal care, policies and procedures and description of the facility were not provided in both of these studies. These details may shed more light on how context influences outcomes and should be investigated in future studies. One recent large, but retrospective, study was performed on 600 selected CS patients in western India. ${ }^{14}$ Similar to our study, they did not find an association between intraoperative complication and type of CS. Although wound infection rates were similar, the incidence of fever and UTI was higher in the emergency CS group. They postulated that this may be more reflective of poor antenatal care in these patients.

Although there was similar maternal morbidity in the two groups, the fetal outcomes were worse in the emergency CS group. This was however not replicated in the recent large study by Gurunule A et al in western India. ${ }^{14}$ This may be due to the overall low incidence of complications, and also they did not compare APGAR scores. The incidence of respiratory distress appeared to be higher in the emergency CS group (7\% versus $4 \%$ ). Najam et al. ${ }^{12}$ in their study from northern India reported a higher number of fetal complications which appeared to be more frequent in the emergency CS group, although this was not statistically evaluated and they did not evaluate APGAR scores. Suwal et al. ${ }^{11}$ in their study from Nepal reported a significantly higher incidence of APGAR scores $\leq 6$ in the emergency CS group. Our results and results from the other studies consistently show poor fetal outcomes for emergency CS group. Whether this is due to fetal distress or complication as an indication for emergency CS or result of emergency CS is not clear and could be evaluated in future studies.
Table 5: Neonatal outcomes

\begin{tabular}{llll}
\hline & Emergency CS & Elective CS & $p$ value \\
\hline $\begin{array}{l}\text { NICU } \\
\text { admission }\end{array}$ & $95(32.5 \%)$ & $34(16.6 \%)$ & $<0.001$ \\
Apgar 1 min & $7(1.4)$ & $8(0.7)$ & $<0.001$ \\
Apgar 5 min & $8.6(0.6)$ & $8.9(0.37)$ & $<0.001$ \\
Weight $(\mathrm{kg})$ & $2.56(0.64)$ & $2.7(0.58)$ & 0.01 \\
Still births & 1 & 0 & - \\
Early neonatal & 2 & 1 & - \\
deaths & & 1 & - \\
Apgar & 4 & & - \\
5 min $\leq 6$ & & 2 & - \\
TTN & 6 & 0 & \\
RDS & 3 & & \\
\hline
\end{tabular}

NICU, neonatal intensive care unit; Apgar, appearance pulse grimace activity respiration score; TTN, transient tachypnoea of new born; RDS, respiratory distress syndrome

\section{LIMITATIONS}

Our study represents a large prospective study, unlike previous retrospective study designs. However, despite large sample size various outcomes related to morbidity and particularly mortality occurred with low frequency, impeding robust statistical interpretation. Unlike some of the previous study, we screened all the deliveries and included all CS performed during the study period. This is likely to reduce sampling bias. However, we had missing data in 48 patients. This is unlikely to impact results, as the studied sample size was larger than the calculated sample size, even after excluding these 48 patients.

\section{CONCLUSION}

The outcomes of emergency versus elective CS vary depending upon the context. The rate of CS at our center is relatively lower, with more emphasis on the trial of vaginal delivery. Although maternal morbidity was similar between the emergency and elective CS patients, the fetal outcomes were worse in the emergency CS patients. Whether this is due to fetal distress or complication as an indication for emergency CS or result of emergency CS is not clear and could be evaluated in future studies.

\section{ACKNOWLEDGMENT}

Authors want to acknowledge Dr Varghese Philip's help in providing the necessary research infrastructure for this project.

\section{REFERENCES}

1. Betrán AP, Ye J, et al. The Increasing Trend in Caesarean Section Rates: Global, Regional and National Estimates: 1990-2014. PLoS One 2016;11(2):e0148343. 
2. Anderson GM. Making sense of rising caesarean section rates: Time to change our goals BMJ 2004;329:696-697

3. Conroy K, Koenig AF, et al. Infectious Morbidity After Cesarean Delivery: 10 Strategies to Reduce Risk. Reviews in Obstetrics and Gynecology 2012;5(2):69-77.

4. Liu S, Liston RM, et al. Maternal mortality and severe morbidity associated with low-risk planned cesarean delivery versus planned vaginal delivery at term. CMAJ 2007; 176(4): 455-460.

5. Amirikia $\mathrm{H}$, Zarewych B, et al. Caesarean section: a 15-year review of changing incidence, indications, and risks. Am J Obstet Gynecol 1981;140(1):81-90.

6. Saha PK, Gulati R, et al. Second stage caesarean section: evaluation of patwardhan technique. Journal of Clinical and Diagnostic Research JCDR 2014;8(1):93-95.

7. Bodian CA, Freedman G, et al. The visual analog scale for painClinical significance in postoperative patients. Anesthesiology: The Journal of the American Society of Anesthesiologists. 2001 Dec 1;95(6):1356-1361.

8. Maternal mortality ratio. http://www.who.int/healthinfo/ statistics/indmaternalmortality/en/. Accessed on 6th May 2015.

9. Shabnam A. Caesarean section delivery in India: causes and concerns. https://iussp.org/sites/default/files/event_call_ for_papers/Caesarean\%20section \%20delivery\%20in $\% 20$ India_0.pdf. Accessed on 6th May 2015.
10. Elvedi-Gasparović V, Klepac-Pulanić T, et al. Maternal and fetal outcome in elective versus emergency caesarean section in a developing country. Coll Antropol 2006;30(1): 113-118.

11. Suwal A, Shrivastava VR, et al. Maternal and fetal outcome in elective versus emergency cesarean section. JNMA J Nepal Med Assoc 2013 Oct-Dec;52(192):563-566.

12. Najam R, Sharma R. Maternal and fetal outcomes in elective and emergency caesarean sections at a teaching hospital in North India. A retrospective study. JARMS 2013;5(1):5-9

13. Daniel S, Viswanathan M, et al. Comparison of fetal outcomes of emergency and elective caesarean sections in a teaching hospital in Kerala. Academic Medical Journal of India 2014;2(1):32-36.

14. Gurunule A, Warke H. Maternal and foetal outcome in elective versus emergency caesarean sections. International Journal of Reproduction, Contraception, Obstetrics and Gynecology 2017;6(4):1222-1228.

15. Raees M, Yasmeen S, et al. Maternal morbidity associated with Emergency versus elective caesarean section. J Postgrad Med Inst 2012;27(1):55-62.

16. Al Nuaim L, Soltan MH, et al. Outcome in elective and emergency cesarean sections: A comparative study. Ann Saudi Med 1996;16(6):645-649. 